\title{
Optimization of the Online Teaching System Based on Streaming Media
}

\author{
Kuiqun Wang $\mathbb{B}$ \\ College of Education Science, Weinan Normal University, Weinan, Shaanxi 714099, China \\ Correspondence should be addressed to Kuiqun Wang; wangkuiqun@wnu.edu.cn
}

Received 8 January 2021; Revised 8 February 2021; Accepted 13 February 2021; Published 23 February 2021

Academic Editor: Wei Wang

Copyright (C) 2021 Kuiqun Wang. This is an open access article distributed under the Creative Commons Attribution License, which permits unrestricted use, distribution, and reproduction in any medium, provided the original work is properly cited.

\begin{abstract}
Network and related network technology limit the traditional online teaching activities, making teaching activities only limited to asynchronous teaching, limiting the advantages of real-time, interactive, and vivid online teaching. As a new online teaching network technology, streaming media technology can realize flexible and efficient two-way communication between teachers and students, simulate virtual face-to-face teaching environment, and produce enough emotional resonance for both sides in the corresponding time and space. In view of the poor communication quality and flexibility of current streaming media technology, this paper will build a selective streaming media online teaching architecture based on animation media service platform. The system innovatively uses DXSDK filtering technology to realize real-time audio acquisition and filtering processing and solves the communication quality problem of streaming media online teaching. Aiming at the flexibility of streaming media online teaching, the system adds online auxiliary teaching function, which can realize online text communication, file information management, and learning tracking interaction between teaching and learning. The experimental part is applied to the online teaching activities of a training institution. From the experimental results, the stability and flexibility of the system are greatly improved within a certain range of users, which has obvious practical value. It has high stability and application value.
\end{abstract}

\section{Introduction}

Online network teaching breaks through the space and time constraints of traditional teaching, which can realize the physical isolation between teachers and students and shift the focus of both teachers and students to the virtual teaching center. Its long-term implementation is conducive to the continuous circulation and promotion of education [1-3]. To some extent, online teaching can also realize the sharing of educational resources, so that excellent educational resources can be fully utilized and recycled. At the same time, it can also promote the continuous cross of various subject resources, so as to further improve the quality of online classroom for teachers and students, which is conducive to the cultivation of complex talents [4-7]. At the same time, the current online teaching is conducive to highlighting the dominant position of students in the classroom, thus forming student-centered classroom activities. However, the current online teaching is subject to the problems of information technology, multimedia technology, and related online teaching concepts, which leads to the lack of communication and flexibility in the current online teaching system. As a result, there are some problems in the traditional online teaching, such as the corresponding teaching quality, teaching methods, students' corresponding learning effects, and the allocation of teachers' teaching resources Due to the obsolete traditional online teaching concept, the effect of traditional online teaching deviates $[8,9]$.

On the optimization strategy of the teaching system under the corresponding streaming media technology, a large number of scientific research institutions have conducted research and analysis on it. Relevant universities in the United States have cooperated with the corresponding network companies to develop the corresponding online course management system, which can realize the release management of any file, provide a platform for knowledge discussion and learning, analyze and test online, and support the corresponding distance learning plan. The establishment 
and online knowledge discussion of such a system promote the improvement of education and teaching system, but its corresponding real-time performance, data transmission quality, and other auxiliary functions are still problems [10-12]; in the relevant university teaching system, the corresponding network multimedia courseware system has been widely used, and the corresponding multimedia technology is mostly text, graphics, video images, and related knowledge. The corresponding multimedia courseware is mostly stored in the corresponding courseware server, which constitutes the multimedia courseware library $[13,14]$; the corresponding network multimedia classroom of the development department of primary and secondary school education group is a centralized online learning platform, which is mainly to establish a classroom-based teaching platform in the central LAN system, and the corresponding system is equipped with a server and contains some students' computers. In the actual teaching process, teachers put the corresponding multimedia courseware into the corresponding network server. Relevant countries in Europe and the United States have applied streaming media technology to online teaching activities for the first time. The corresponding streaming media technology is divided into two categories [15-18]: sequential streaming media technology and real-time streaming media technology. The main difference lies in the differences between the corresponding servers. At the same time, there are serious problems in the corresponding transmission protocol layer. However, no matter what form streaming media, its corresponding structure is different and relatively fixed: collector, encoder, transmission channel, memory, and the corresponding decoder, and the current streaming media used in network teaching programs mainly include real media technology, rapid integration technology, and Microsoft Windows Media Technology. These technologies have different advantages at different levels, but they all need to adapt to the corresponding fixed hardware system. Microsoft Windows Media Technology is most suitable for small educational institutions, but there are serious communication quality problems, and its corresponding auxiliary functions are relatively few [19-23].

Based on the above discussion, it is necessary to develop an online teaching system with high communication quality and certain auxiliary online teaching function. This paper will build a selective streaming media online teaching architecture system based on animation media service platform. The system innovatively uses DXSDK filtering technology [24-28] to realize the real-time audio acquisition and filtering processing, so as to solve the communication quality problem of streaming media online teaching. As for the flexibility of streaming media online teaching, the system adds online auxiliary teaching function, which can realize online text communication, file information management between teaching and learning, and learning tracking interaction during online teaching. The experimental part of the system is applied to the online teaching activities of a training institution. The experimental results show that the stability and flexibility of the system are greatly improved under a certain range of users, and it has obvious practical value.
The corresponding structure of this paper is as follows: the second section will focus on the streaming media technology architecture used in this paper and focus on the analysis of the DXSDK filtering technology module and the corresponding online auxiliary function module; the third section of this paper will carry out experiments based on the system designed in this paper and analyze the experimental data; finally, it will summarize and prospect this paper.

\section{Analysis and Research of the Online Teaching Architecture System Based on Selective Streaming Media}

In this paper, the top-level design of the system is mainly based on the selective streaming media online teaching architecture. The corresponding architecture block diagram is shown in Figure 1. The whole architecture is composed of four parts: media encoder, media file data memory, online teaching media server, and corresponding media player. The four corresponding parts cooperate to form the whole streaming media service system's unification. From the corresponding structure diagram in Figure 1, the details of the corresponding four parts are as follows: the media online encoder mainly realizes the production of the original media files and the real-time media data collected by the corresponding host and guest cameras, so as to form the corresponding file format suitable for network transmission; the online media file memory mainly uses the media files; the media player in the corresponding media service is mainly used to receive network media-related data and play it locally.

2.1. Analysis of Streaming Media Technology Selection. The main technical points of the selective streaming media technology architecture are the establishment of the communication model of the selective streaming media online teaching architecture; the establishment of the connection object; the establishment of the connection data source model.

In the establishment of the communication model of the corresponding selective streaming media online teaching architecture, we mainly consider the establishment of the data stream and the corresponding shared objects. The data stream corresponding to the selective streaming media technology and the corresponding shared host object is based on the $\mathrm{C} / \mathrm{S}$ basic mode. The corresponding main system architecture server mainly realizes the data transmission, and the corresponding transmission is mainly sent through the client request. The data server queries and processes the data and returns the corresponding processing results to the client. In this process, we need to keep the data connection and transmission. The corresponding communication model of the selective streaming media online teaching architecture is shown in Figure 2. It is mainly based on time. It can realize the synchronous transmission and processing of audio, video, and other interactive data at the same time. At the same time, the corresponding data stream can realize simple data publishing and information subscription processing. In this selective streaming media 


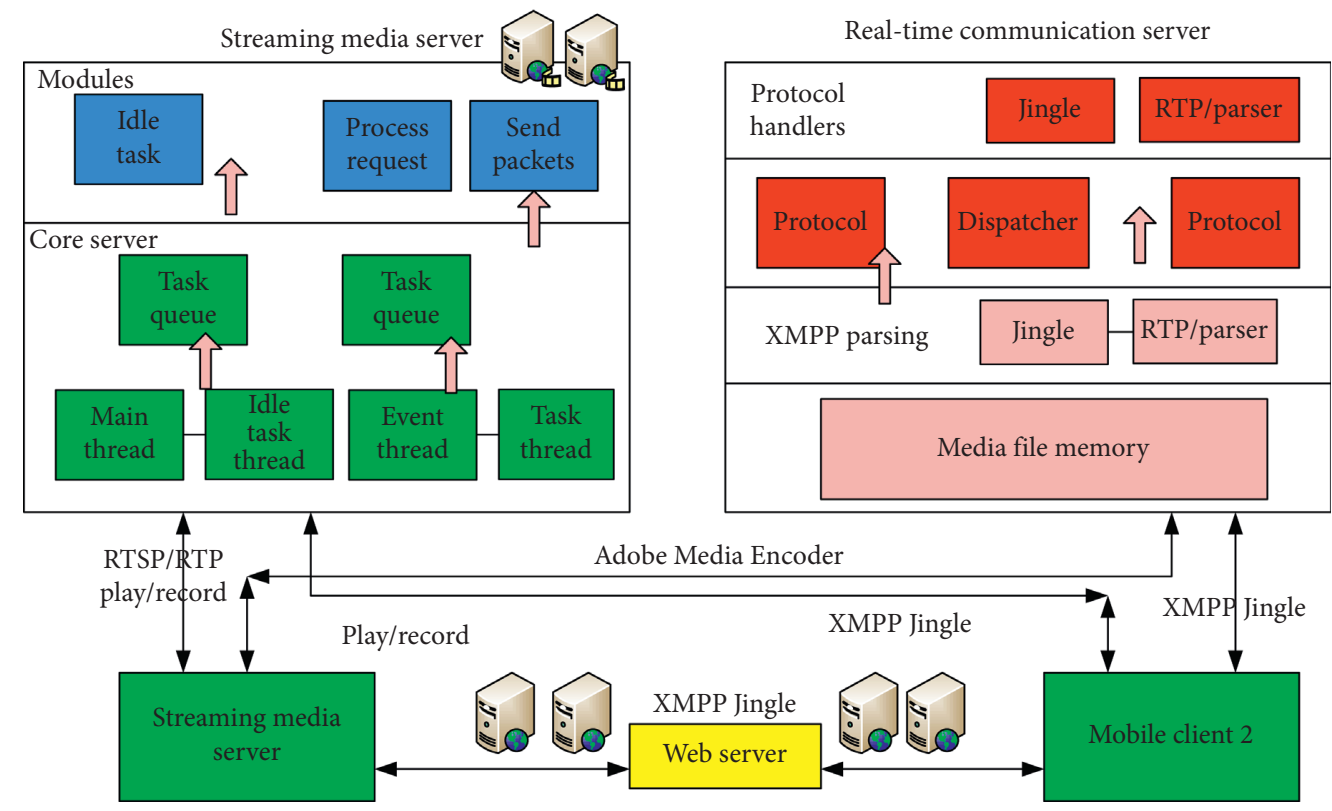

FIgURE 1: Framework of selective streaming media online teaching system.

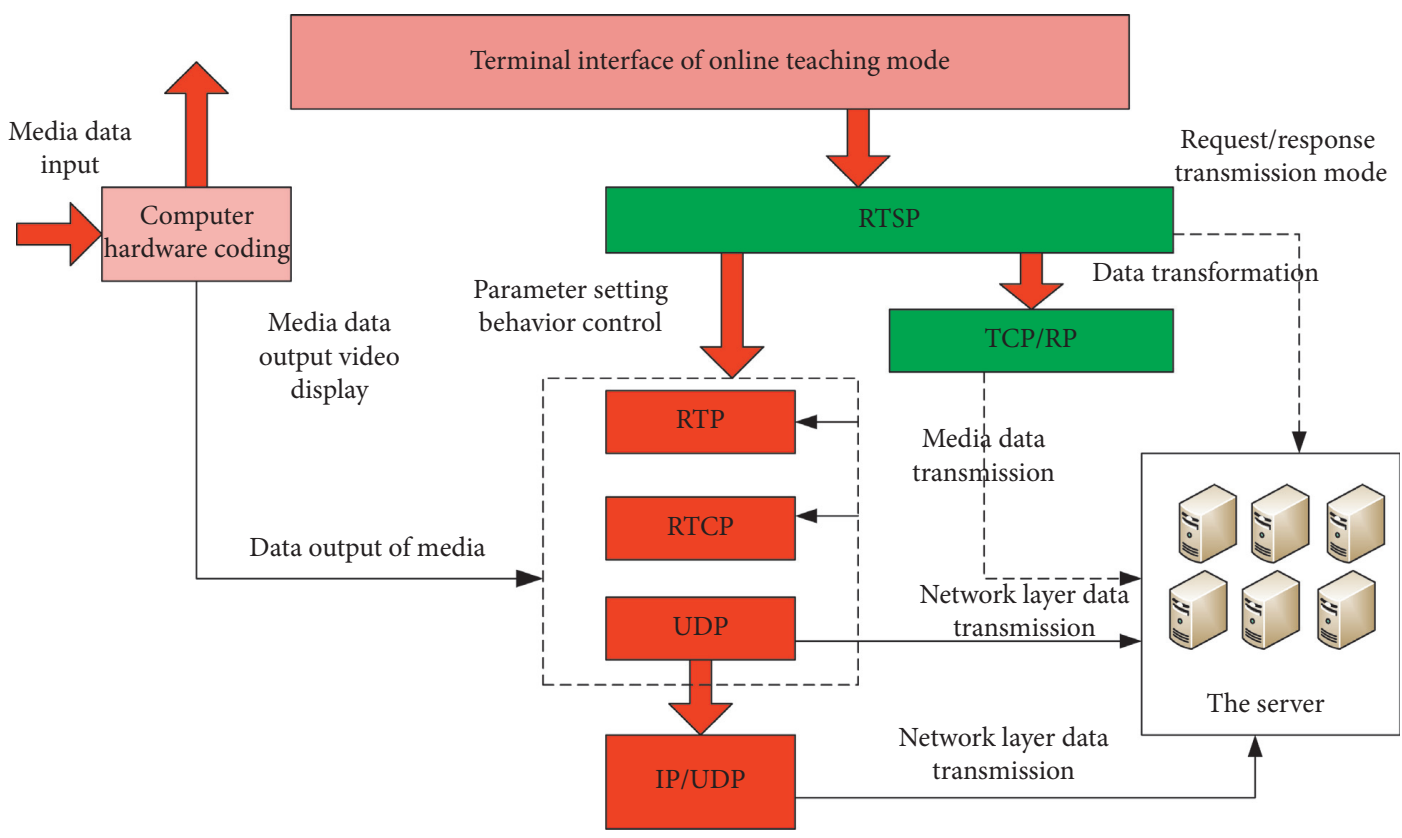

\footnotetext{
$\Longrightarrow$ Interprogramming interface transfer

$\longrightarrow$ The dotted line is streaming media

$---\rightarrow$ The real line is IP network
}

FIGURE 2: Communication model of selective streaming media online teaching architecture.

architecture, the corresponding data are based on a file in the form of sh. In the architecture, the corresponding sharing objects are mainly divided into two levels: local sharing and remote sharing. The corresponding local sharing is that the user main body stores the local computer or multimedia related data and realizes the local sharing. The corresponding remote sharing is mainly to provide the corresponding information and data synchronous sharing. At the same time, it also needs to realize remote data sharing. It plays the main role of real-time sharing. The shared subject and object in the selective streaming media online teaching architecture can realize data sharing among multiple users and realize all kinds of connection of synchronous information. 
At the level of establishing connection object, it mainly realizes the corresponding data connection, and at the same time, it also depends on the important function of sharing subject object and object object. Its corresponding connection object establishment framework is shown in Figure 3. In the process of establishing connection object, we need to focus on three principles: classification principle, information priority principle, and control priority principle. In the corresponding classification principle level, it mainly deals with the relevant real-time information stream and strictly divides the corresponding information into data sets, video sets, and audio sets. The corresponding database is like a storage data container, and it must be clear which related files have been processed and which have not been processed. In the information priority principle level, it mainly focuses on the analysis of the priority of related information. The corresponding priority order is the lowest video and the highest data packet. When the corresponding data object is connected, it is necessary to consider the corresponding reason when the priority of the data packet is abandoned. Once the related data of the data packet is discarded, the corresponding user client may not be able to obtain the corresponding logic and operation information. If we consider the priority of the abandoned packets, it is impossible to change the priority order under the existing principles, but it is feasible to change the corresponding data requirements of the client in this passive situation.

In the connection data source model building level, it mainly considers the subject and object information, data information, course information, teaching information, use information, and so on. The corresponding data source model is shown in Figure 4, from which we can see that it mainly follows the promotion of three tasks:

(1) The corresponding flash terminal realizes the realtime transmission of the corresponding client requests to the corresponding architecture server, and the architecture server carries out the relevant processing and analysis

(2) Translate the corresponding request and data packet of the corresponding flash client, and form the request and data of the server with the translated content

(3) Translate the corresponding response and data of the architecture server into the corresponding local data type.

2.2. DXSDK Filtering Technology Module Analysis. The corresponding architecture of the filter module used in this paper is shown in Figure 5. The corresponding filter mainly uses three types of filters: source filter, conversion filter, and corresponding performance filter. The corresponding filter in this structure is mainly responsible for the corresponding data acquisition, and the corresponding data source can be file and digital camera. The filter will continue to transmit the data collected by the corresponding filter. The corresponding filter also needs to be responsible for the continued downward transmission of the corresponding data, separation/synthesis, and decoding/encoding of the corresponding main data stream. After such data processing, the corresponding data will be transmitted to the data graphics card and sound card. Finally, the corresponding multimedia presentation is output to a file for transmission.

At the corresponding acquisition filter level, it is mainly implemented on PC. As can be seen from Figure 5, the corresponding filtering process is as follows:

(1) Use the corresponding function to create the corresponding system enumeration component object, and obtain the corresponding interface

(2) Create the corresponding enumerator for the specified data type directory based on the above interface, and obtain the corresponding interface. The corresponding types here are video input type and audio input type.

(3) Enumerate the identification of different devices in the corresponding type directory on the corresponding interface, and implement the corresponding function interface on each device identification.

The detailed structure of the corresponding filter is shown in Figure 6. The corresponding architecture in this paper mainly encodes the corresponding input video and audio signals into the corresponding ASF format coding. After the system architecture creates the corresponding compression filter, the corresponding generator is created in the corresponding internal using the corresponding function, so that the coding can be based on this. From the corresponding Figure 6, we can see that the generator itself is not responsible for encoding the corresponding final destination but sends this task to the receiver object.

\subsection{Research on the Design of Online Auxiliary Function} Module. In addition to the above-related filter level innovation, this paper also proposes the design of the corresponding online auxiliary function module; its main online auxiliary functions include online text communication and the establishment of the corresponding virtual classroom. In the corresponding level of text communication, it is mainly to realize online text communication, so as to realize the text communication platform between teachers and students. In the corresponding level of text information transmission, it is mainly based on Winsock technology. After the connection between teachers and students, it mainly uses the corresponding socket for message transmission. There are three types of corresponding sockets, streaming socket, streaming socket, and datagram socket, and corresponding original socket. The corresponding streaming socket provides bidirectional, orderly, nonrepetitive, and nonrecord boundary data stream service, which is mainly used for processing a large number of data, and it is mainly connection-oriented, so corresponding data transmission link must be established, and the transmitted data must be verified to ensure the 


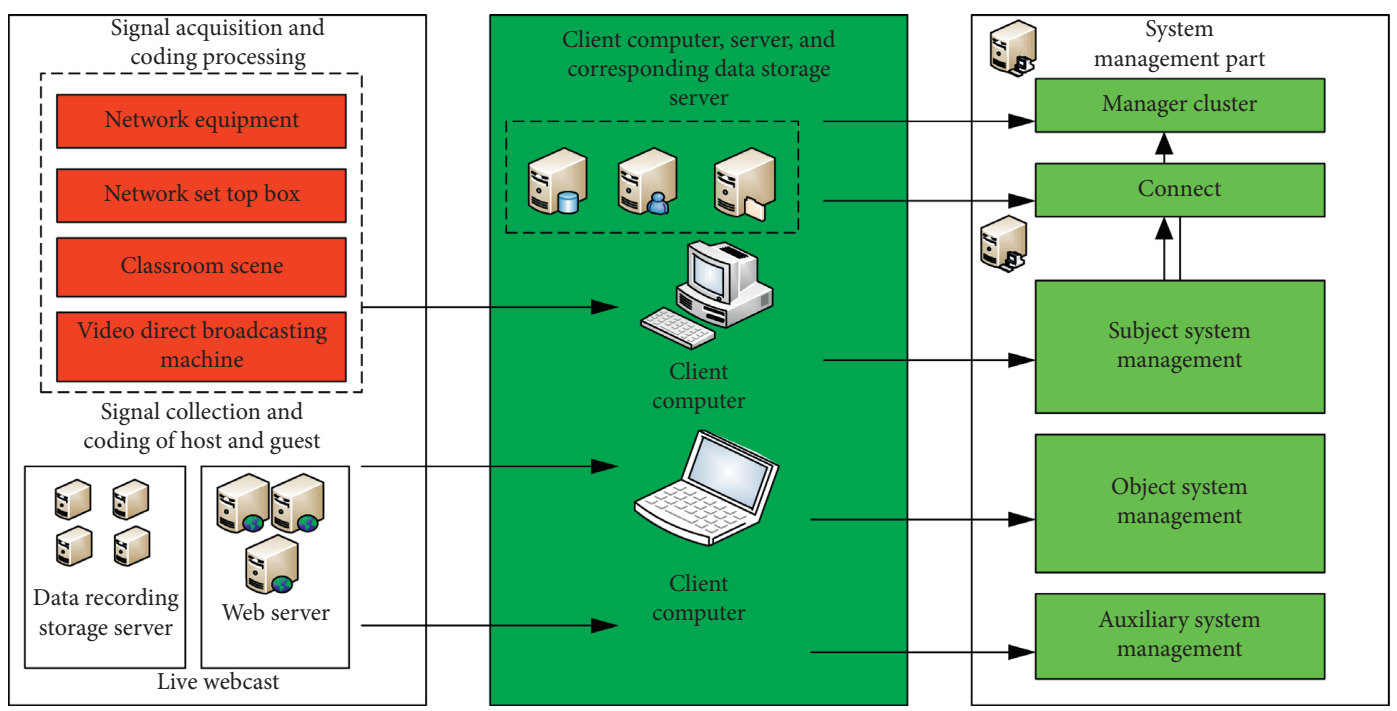

FIGURE 3: Framework diagram of connection object establishment of selective streaming media online teaching architecture.

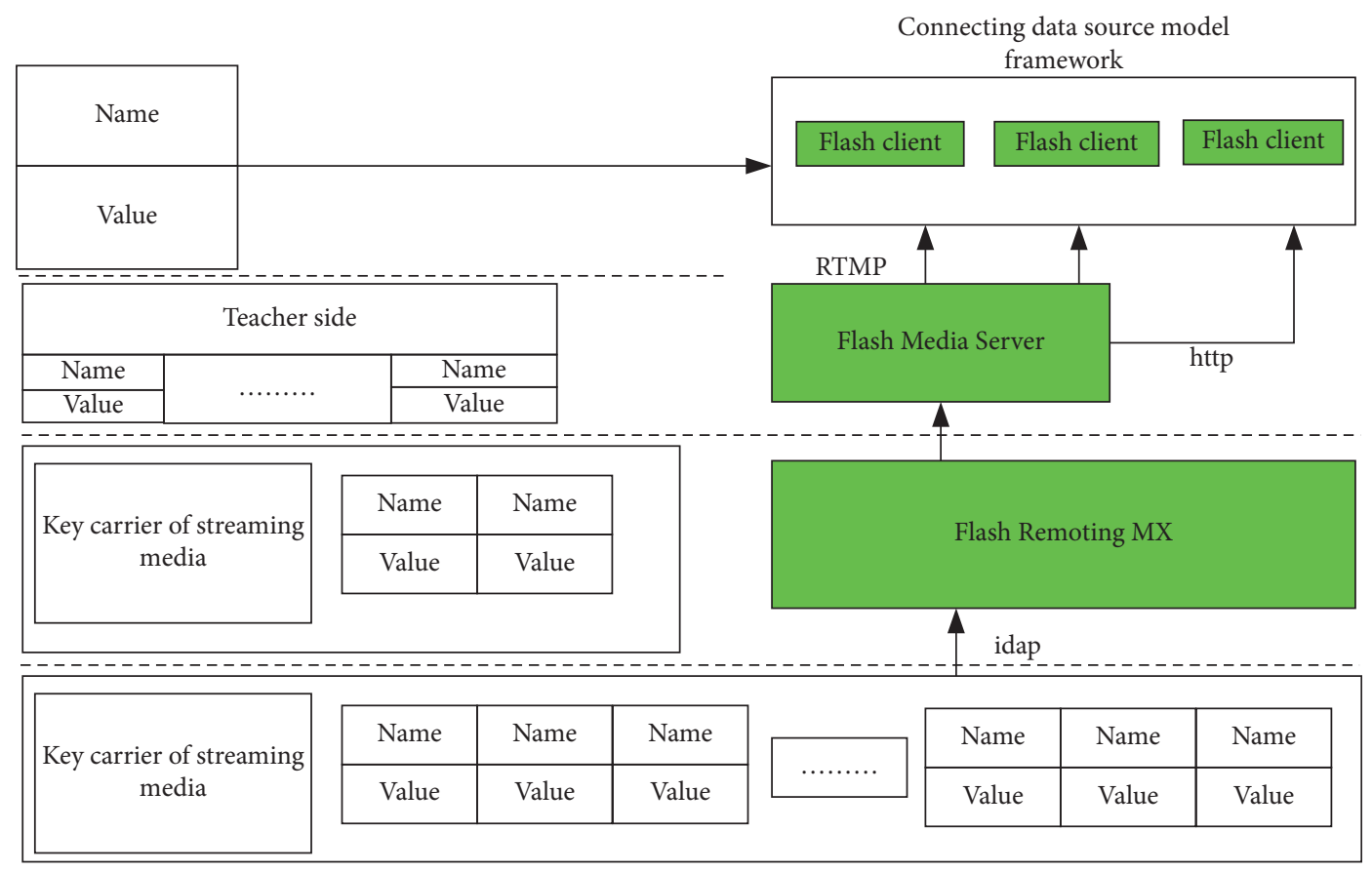

FIGURE 4: Framework of connection data source model.

accuracy of data; datagram socket mainly realizes bidirectional data transmission, but it cannot guarantee the accuracy of the whole data, but it retains the record boundary of the relevant data; in the corresponding original socket level, it mainly saves the IP address header of the complete data in the data packet, which can realize the analysis and research of the original socket on the data. In the corresponding auxiliary function of online text communication, it is mainly implemented by a thread. The corresponding thread is mainly two threads, which are, respectively, thread 1 and thread 2 . The corresponding thread 1 is mainly implemented by teachers. When teachers join the virtual classroom, they realize the socket establishment, binding, and corresponding monitoring work. The corresponding thread 2 is mainly to realize the information between students. The corresponding student object corresponds to a socket, the corresponding socket is saved in the corresponding socket group according to the sequence, and the corresponding teacher realizes the corresponding sending and receiving information in turn. The corresponding thread of online text communication is shown in Figure 7.

In the corresponding virtual classroom creation level, it is mainly to create the corresponding $3 \mathrm{D}$ virtual environment, so as to realize the simulation of the corresponding real environment. The corresponding virtual 


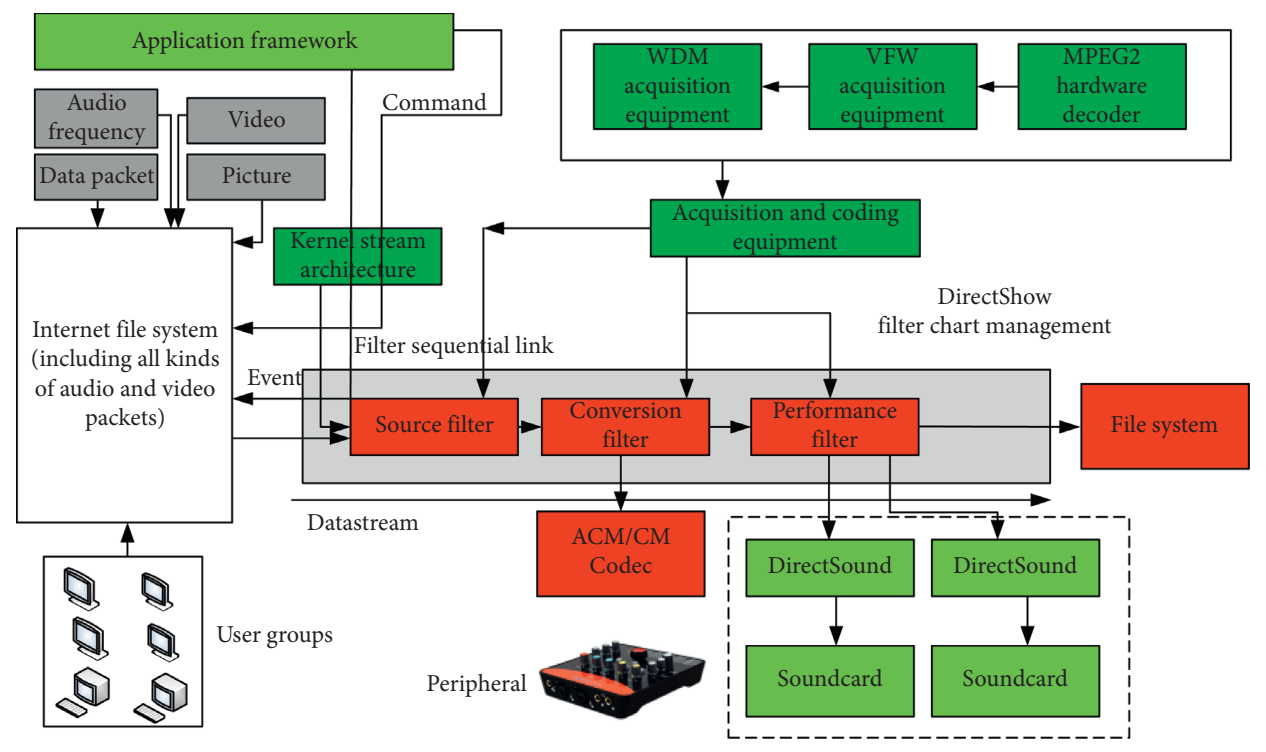

FIgURE 5: Architecture diagram of a filter module.

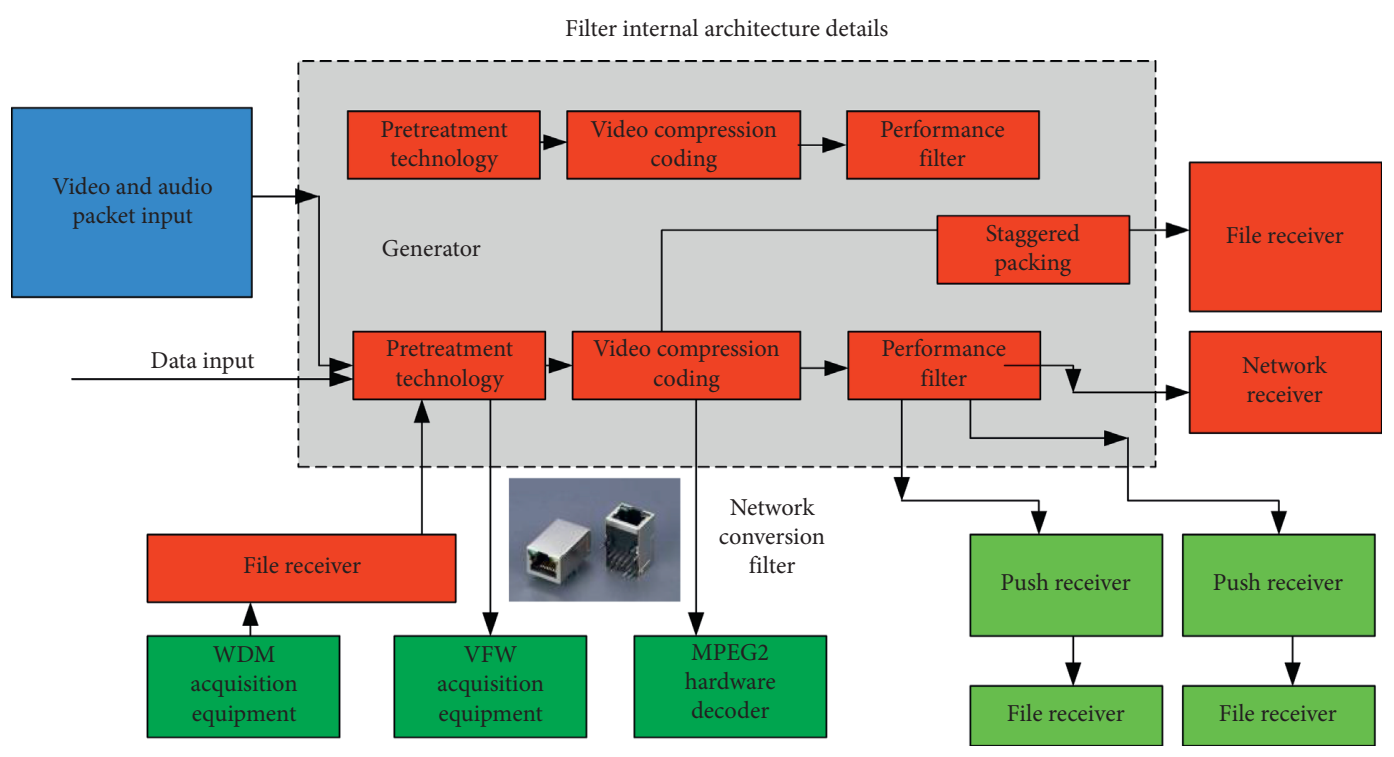

Figure 6: Detailed structure of the filter.

classroom member list is mainly used to present the list of students currently participating in text communication, so as to facilitate the management and viewing of the corresponding teachers. When the corresponding students join, the teacher can pass the previous list of students who have joined the virtual classroom to the corresponding new students and at the same time distribute the address of the corresponding new students to other existing students, so as to ensure that the corresponding information of the virtual classroom can be realized synchronously and at the same time arrange the relevant information according to the order of entering. When the corresponding students exit, the main exit is divided into exit system and exit communication. The corresponding exit system mainly refers to that the students close the whole online education system directly. The corresponding exit communication mainly refers to that the students leave the corresponding virtual classroom temporarily but do not do the corresponding text communication. In the virtual classroom, no matter what type of students' exit, the main reason is that the students leave the virtual classroom. When the corresponding student exits, it means that the communication socket is closed, and the corresponding information will be displayed on the corresponding teacher. At this time, the corresponding teacher should delete the corresponding student address in the virtual classroom list and inform other corresponding students. 


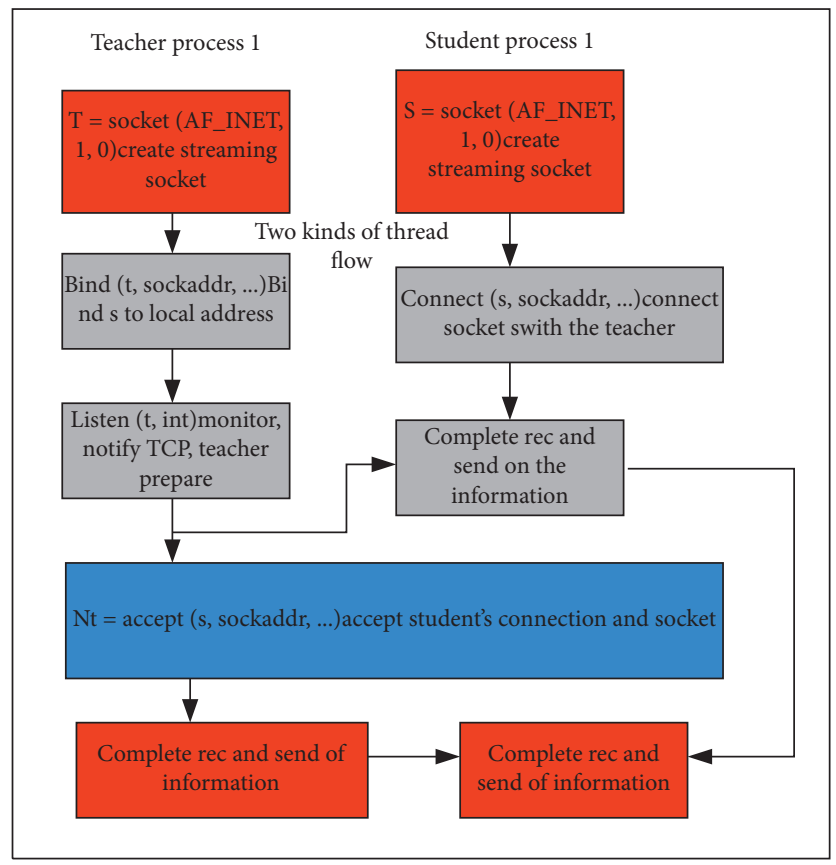

FIgURE 7: Thread diagram of line text communication.

\section{System Test and Result Analysis}

In the corresponding system test level, the main test content is as follows: $\mathrm{C} / \mathrm{S}$ mode video distribution test and video distribution test. In the corresponding $\mathrm{C} / \mathrm{S}$ mode video distribution test level, the corresponding test interface is shown in Figure 8, and the corresponding video distribution test content is also shown in the corresponding Figure 8 test interface.

In the corresponding system failure rate (such as online, offline, network data information blocking, information transmission failure, and display failure) level, this paper compares it with the traditional online teaching mode, and the corresponding comparison curve is shown in Figure 9. From the figure, it can be seen that the corresponding online teaching mode in this paper has a lower failure rate and higher efficiency than the traditional mode.

The host computer and the corresponding server used in the selective online teaching system architecture used in this paper are ordinary microcomputer systems. When the corresponding amount of data increases, the corresponding online mode will show the problem of jam or even play delay. The $\mathrm{C} / \mathrm{S}$ video distribution mode used in this paper can realize the problem of video fluency when the amount of data is large. At the same time, the corresponding text communication, member list, and preview functions are verified in the actual test. The experimental results show that the corresponding results meet the actual needs, and the corresponding flexibility is relatively strong. The corresponding online text communication test list is shown in Table 1. It can be seen from the table that the corresponding login IP number, chat room number, number of people in chat room, and other pieces of relevant basic information can be displayed in real time.

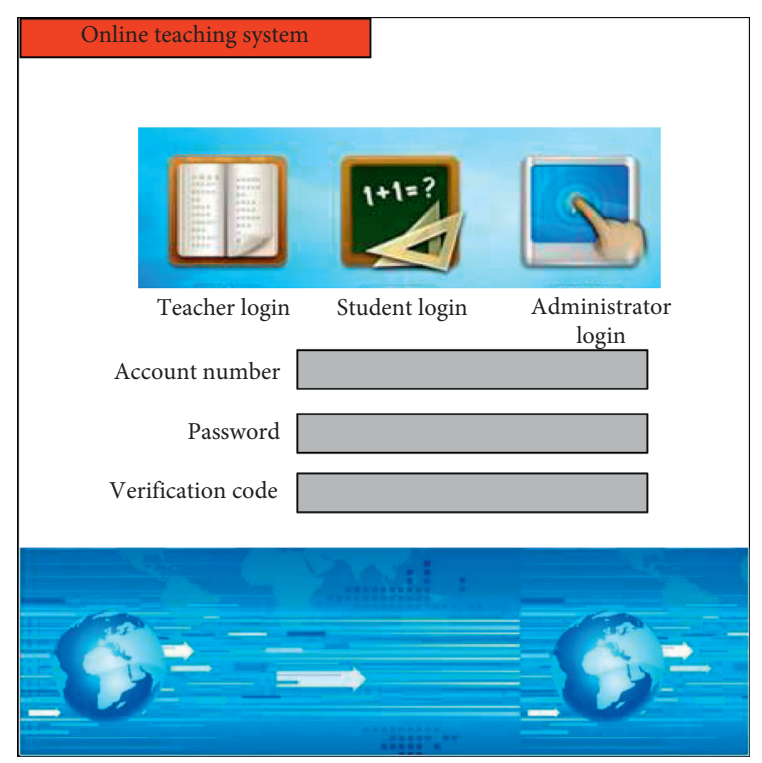

Figure 8: Test interface.

In this paper, the corresponding code implementation level is mainly implemented by the relevant assembly language; this paper gives the corresponding source code file when the audio transmission in the audio transmission part is mainly composed of the video box and the corresponding release box; when the corresponding customer body sends the corresponding requirements, the corresponding requirements will be passed, and the real-time stream will transmit the corresponding video and audio files and send them to the corresponding FMS module, and the corresponding local video box will be displayed. 


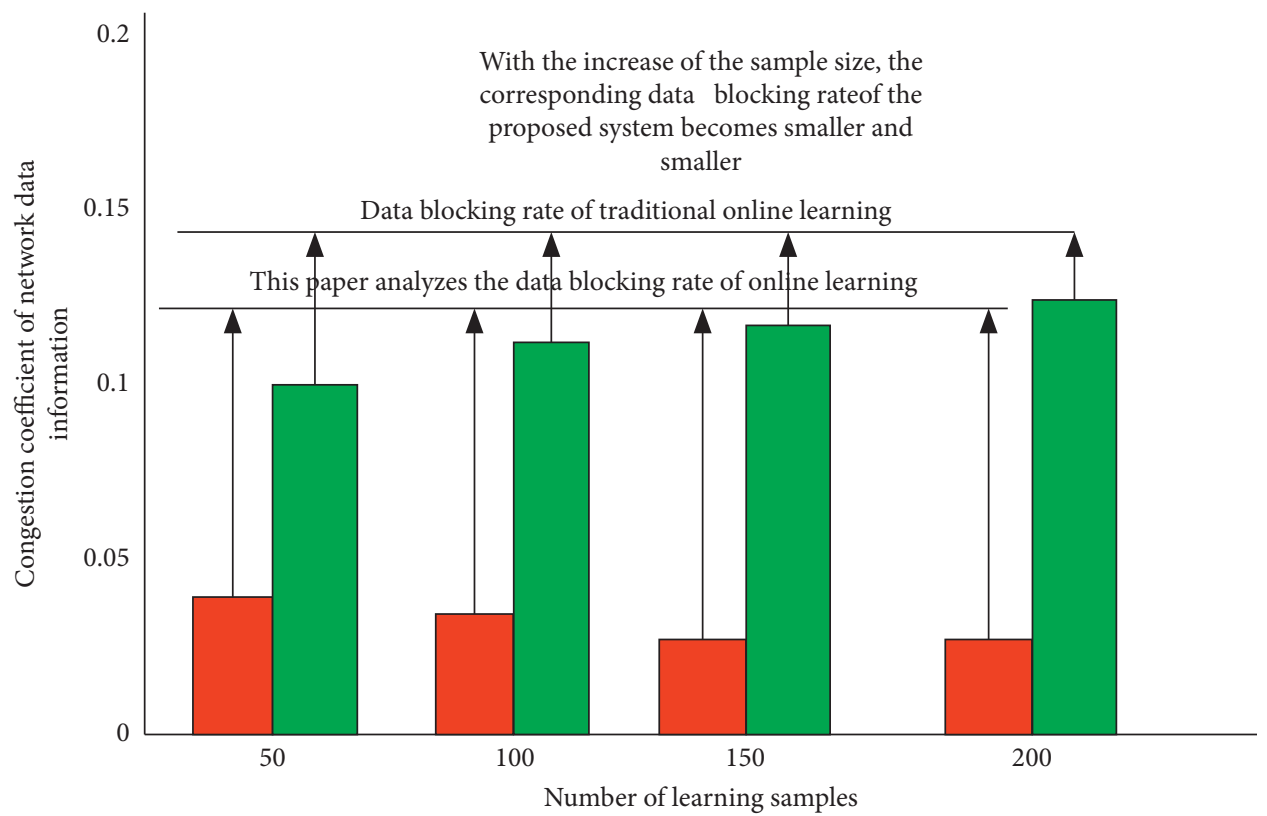

(a)

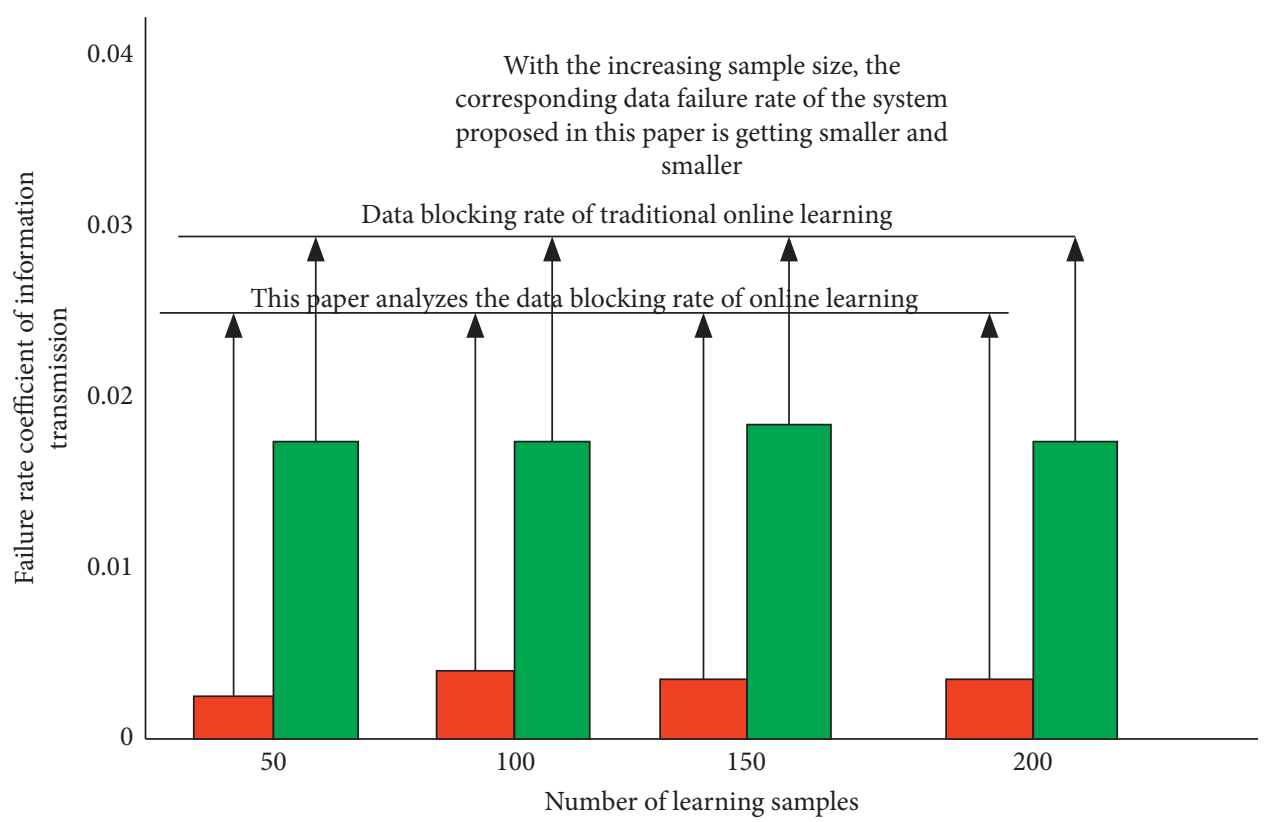

(b)

FIgURE 9: Continued. 


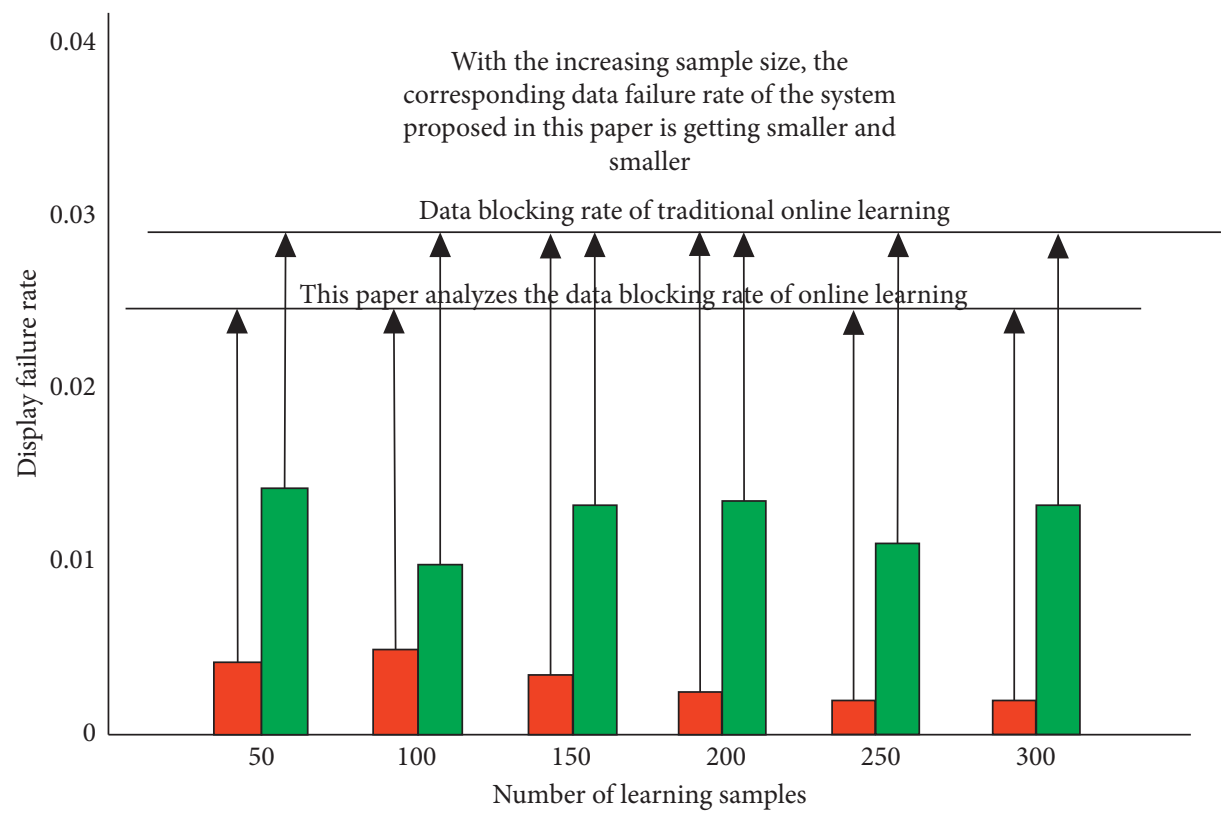

(c)

Figure 9: (a) Comparison chart of online teaching fault curve. (b) Comparison chart of online teaching fault curve. (c) Comparison chart of online teaching fault curve.

TABLE 1: Details of online text communication.

\begin{tabular}{|c|c|c|c|c|}
\hline Field name & Corresponding data type and length & Keyword & Is it not empty & Field meaning \\
\hline LoginRecordID & Int $(4)$ & $\mathrm{Y}$ & $\mathrm{N}$ & Login record number \\
\hline ChatroomID & Int (4) & $\mathrm{N}$ & $\mathrm{Y}$ & Chat room number \\
\hline UserLoginTime & Datetime & $\mathrm{N}$ & $\mathrm{Y}$ & User login time \\
\hline UserOutTime & Datetime & $\mathrm{N}$ & $\mathrm{Y}$ & User logout time \\
\hline ClassRecordID & Nchar (50) & $\mathrm{N}$ & $\mathrm{Y}$ & Record number \\
\hline UserCount & Int $(4)$ & $\mathrm{N}$ & $\mathrm{Y}$ & Landing number \\
\hline Login IP & Nchar (50) & $\mathrm{N}$ & $\mathrm{Y}$ & Record number \\
\hline Data type & Nchar (50) & $\mathrm{N}$ & $\mathrm{Y}$ & Record number \\
\hline Data content & Datetime & $\mathrm{N}$ & $\mathrm{Y}$ & User logout time \\
\hline T_ChatroomID & Int $(4)$ & $\mathrm{N}$ & $\mathrm{Y}$ & Chat room number \\
\hline
\end{tabular}

\section{//Set up microphone}

teacherMic $=$ teacherphone.get ();

teacherMic.useSuppression (1);

teacherMic.setSilenceLevel (0);

//Set up the corresponding camera

teacherCamera $=$ Camera.get () ;

teacherCamera. motionalevel $=45$;

teacherCamera.quality $=80$;

teacherCamera.setquality $(0,80)$;

teacherCamera.setMode $(355,405,10)$;

//Connect the corresponding microphone and camera to the real-time stream

After the actual system testing and verification, the selective online teaching system proposed in this paper has obvious advantages in improving the efficiency between teachers and students and the corresponding functional satisfaction compared with the traditional online teaching method. At the same time, it has obvious advantages in the energy consumption and resource utilization of the source code. In the actual test, the corresponding filter level lacks the corresponding connectivity, and the running in between the corresponding systems still has problems. This paper will focus on the connectivity and systematicness of the filter in the follow-up research.

\section{Conclusion}

This paper mainly analyzes the problems existing in the corresponding architecture of the current online teaching, analyzes the advantages and disadvantages of the corresponding online teaching strategies and platforms, and points out the problems existing in the corresponding architecture. In order to solve the problem of online teaching quality based on DXSDK, this paper builds an online teaching platform for streaming media filtering. As for the flexibility of streaming media online teaching, the system adds online auxiliary teaching function, which can realize 
online text communication, file information management between teaching and learning, and learning tracking interaction during online teaching. The experimental part of the system is applied to the online teaching activities of a training institution. The experimental results show that the stability and flexibility of the system are greatly improved under a certain range of users, and it has obvious practical value. In the follow-up research, this paper will focus on the application of the system in large educational institutions and optimize its experiment.

\section{Data Availability}

The data used to support the findings of this study are available from the corresponding author upon request.

\section{Conflicts of Interest}

The author declares that there are no conflicts of interest reported in this paper.

\section{References}

[1] Y. Hu and Q. Yang, "A new scheduling mechanism of BitTorrent streaming system based on improved PSO algorithm," Journal of Computers, vol. 54, no. 6, pp. 32-44, 2015.

[2] M. M. Elkholy and A. Fathy, "Optimization of a PV fed water pumping system without storage based on teaching-learning-based optimization algorithm and artificial neural network," Solar Energy, vol. 139, no. 1, pp. 199-212, 2016.

[3] A. H. Zahran and C. J. Sreenan, "Threshold-based media streaming optimization for heterogeneous wireless networks," IEEE Transactions on Mobile Computing, vol. 9, no. 6, pp. 753-764, 2010.

[4] S. Miyata, T. Murase, and K. Yamaoka, "Novel access-point selection for user QoS and system optimization based on user cooperative moving," IEICE Transactions on Communications, vol. E95.B, no. 6, pp. 931-935, 2011.

[5] V. Martín, J. Cabrera, and N. García, "Design, optimization and evaluation of a $q$-learning HTTP adaptive streaming client," IEEE Transactions on Consumer Electronics, vol. 62, no. 4, pp. 380-388, 2016.

[6] K. Ren, G. Bal, and A. H. Hielscher, "Frequency domain optical tomography based on the equation of radiative transfer," SIAM Journal Scientific Computing, vol. 28, no. 4, pp. 1463-1489, 2015.

[7] S. Seo, D. E. Ko, and J. M. Chung, "Combined time bound optimization of control, communication, and data processing for FSO-based 6G UAV aerial networks," ETRI Journal, vol. 42, no. 5, pp. 700-711, 2020.

[8] A. H. Payberah, J. Dowling, F. Rahimain et al., "Distributed optimization of P2P live streaming overlays," Computing, vol. 94, no. 8-10, pp. 621-647, 2012.

[9] J. Xu, K. Takagi, and S. Sakazawa, "Motion synthesis for synchronizing with streaming music by segment-based search on metadata motion graphs," Scientia Horticulturae, vol. 130, no. 4, pp. 1-6, 2011.

[10] C. H. Lin, P. C. Hsiu, and C. K. Hsieh, "Dynamic backlight scaling optimization: a cloud-based energy-saving service for mobile streaming applications," IEEE Transactions on Computers, vol. 63, no. 2, pp. 335-348, 2014.

[11] W.-S. Lim, D.-W. Kim, and Y.-J. Suh, "Design of efficient multicast protocol for IEEE 802.11n WLANs and cross-layer optimization for scalable video streaming," IEEE Transactions on Mobile Computing, vol. 11, no. 5, pp. 780-792, 2012.

[12] M. Claeys, S. Latré, J. Famaey, T. Wu, W. Van Leekwijck, and F. De Turck, "Design and optimisation of a (FA)Q-learning-based HTTP adaptive streaming client," Connection Science, vol. 26, no. 1, pp. 25-43, 2014.

[13] A. Koppel, B. M. Sadler, and A. Ribeiro, "Proximity without consensus in online multiagent optimization," IEEE Transactions on Signal Processing, vol. 65, no. 12, pp. 3062-3077, 2017.

[14] Y. Gu and C. Q. Wu, "Performance analysis and optimization of distributed workflows in heterogeneous network environments," IEEE Transactions on Computers, vol. 65, no. 4, pp. 1246-1262, 2016.

[15] M. Liu, F. R. Yu, Y. Teng, V. C. M. Leung, and M. Song, "Distributed resource allocation in blockchain-based video streaming systems with mobile edge computing," IEEE Transactions on Wireless Communications, vol. 18, no. 1, pp. 695-708, 2019.

[16] L. Vigneri, T. Spyropoulos, and C. Barakat, "Low cost video streaming through mobile edge caching: modelling and optimization," IEEE Transactions on Mobile Computing, vol. 18, no. 6, pp. 1302-1315, 2019.

[17] T. Huang, Q.-F. Cao, J. Liu et al., "A multi objective optimization design tool for traveling-wave tubes' electron optics system," IEEE Transactions on Electron Devices, vol. 66, no. 4, pp. 1965-1970, 2019.

[18] G. Cheung, A. Ortega, and N.-M. Cheung, "Interactive streaming of stored multiview video using redundant frame structures," IEEE Transactions on Image Processing, vol. 20, no. 3, pp. 744-761, 2011.

[19] J. Wu, C. Yuen, N.-M. Cheung, J. Chen, and C. W. Chen, "Modeling and optimization of high frame rate video transmission over wireless networks," IEEE Transactions on Wireless Communications, vol. 15, no. 4, pp. 2713-2726, 2016.

[20] J. Yang, B. Yang, S. Chen, Y. Zhang, Y. Zhang, and L. Hanzo, "Dynamic resource allocation for streaming scalable videos in SDN-aided dense small-cell networks," IEEE Transactions on Communications, vol. 67, no. 3, pp. 2114-2129, 2019.

[21] K. Yu, L. While, M. Reynolds et al., "Multiobjective optimization of ethylene cracking furnace system using self-adaptive multiobjective teaching-learning-based optimization," Energy, vol. 148, no. 3, pp. 469-481, 2018.

[22] W. Liu and R. Mohan, "SU-E-T-625: robustness evaluation and robust optimization of IMPT plans based on per-voxel standard deviation of dose distributions," Medical Physics, vol. 39, no. 6, p. 3850, 2012.

[23] S. K. Perepu and A. K. Tangirala, "Online estimation of missing data using sparse optimization techniques with applications to classical control," IEEE Transactions on Control Systems Technology, vol. 27, no. 2, pp. 495-506, 2019.

[24] U. Yalcin, A. D. Karaoglan, and I. Korkut, "Optimization of cutting parameters in face milling with neural networks and taguchi based on cutting force, surface roughness and temperatures," International Journal of Production Research, vol. 51, no. 11-12, pp. 3404-3414, 2013.

[25] H. Boudjefdjouf, R. Mehasni, A. Orlandi, H. R. E. H. Bouchekara, F. de Paulis, and M. K. Smail, "Diagnosis of multiple wiring faults using time-domain reflectometry and teaching-learning-based optimization,” Electromagnetics, vol. 35, no. 1, pp. 10-24, 2015.

[26] O. M. Silva and M. M. Neves, "A strategy based on the strainto-kinetic energy ratio to ensure stability and convergence in topology optimization of globally resonating one-material 
structures," International Journal for Numerical Methods in Engineering, vol. 121, no. 16, pp. 3636-3659, 2020.

[27] V. Nilsen, E. Christensen, M. Myrmel, and A. Heistad, "Spatio-temporal dynamics of virus and bacteria removal in dual-media contact-filtration for drinking water," Water Research, vol. 156, pp. 9-22, 2019.

[28] W. Zhang, S. Zhang, S. Guo, Y. Yang, and Y. Chen, "Concurrent optimal allocation of distributed manufacturing resources using extended teaching-learning-based optimization," International Journal of Production Research, vol. 55, no. 3, pp. 718-735, 2017. 